Available online @ https://jiem.jnnce.ac.in https:www.doi.org/10.37314/JJEM.2020.040208 Indexed in International Scientific Indiexing (ISI) Impact factor: 1.025 for 2018-19 Published on: 30 March 2020

\title{
Back Propagation Classification of EEG for Brain-Computer Interface
}

\author{
Rajashekhar $\mathrm{U}^{1}$, Neelappa ${ }^{2}$, Rashmi M. Hullamani ${ }^{3}$ \\ Bhagyamma $\mathrm{S}^{4}$, Pavithra $\mathrm{PS}^{5}$, Choodarathnakara $\mathrm{A} \mathrm{L}^{6}$ \\ ${ }^{1 *, 2,4,5,6}$ Department of Electronics and Communication Engineering, \\ Govt. Engineering College, Kushalnagar, India, \\ ${ }^{3}$ Department of Electronics and Telecommunication Engineering \\ JNN College of Engineering, Shimoga, India
}

uraju54@gmail.com,neel.m.dy@gmail.com,rashmimh@jnnce.ac.in, bhagya.gec@gmail.com pavithrakumarps@gmail.com, choodarathnakara@gmail.com

\begin{abstract}
The diagnosis system presents to classify the Electroencephalogram (EEG) brain signal of patient to distinguish between normal and abnormal which are tumor and epilepsy with better classification accuracy. To design automated classification of EEG signals for the detection of normal and abnormal activities using Wavelet transform and Artificial Neural Network (ANN) Classifier is considered. Here, the system uses the back propagation with feed forward for classification which follows the ANN classification with data set training. For training, the statistical principal features will be extracted with facilitate of data base samples. The test sample is going to be classified using ANN classifier parameters and its features. The system gives better performance accuracy for different test samples
\end{abstract}

Keywords: Artificial Neural Network, Brain-Computer Interface, Electroencephalogram, Support Vector Machine

\section{Introduction}

A Brain-Computer interface (BCI) is a one of the communication between human brain and an external device. It is also called neural-control interface, or brain machine interface. Such system is allowing people to communicate through measurements of brain activity, without requiring any movement. It helps paralyzed people solve their problem and make them to communicate, based on the brain sense which detects blink from eye. Brain to chosen litter and make a sentence to communicate with others. BCI is collecting the electrical signals by brain and converting into a control signals to different applications like a keyboard/mouse, robotic, wheelchairs etc. Cashero and Anderson [1] focused on the p300 speller paradigm and the identification of classification accuracies using Blind source separation method (BSS). It consist of three methods

ICA, MNF or PCA these method improves the classification accuracy is $85 \%$ over the original data. Pham et al., [2] developed a unified classification scheme based on ensemble classifier and adaptive learning. Here using SVM method, the experimental result increasing speed and accuracy 75 to $91.26 \%$ with 12.65 iterations. Hoffmann et al.,[3] proposed many interesting application of BCI and how Automatic relevance determination(ARD) and result comparing with two technique that is SBDA and SVM. SVM gives the best accuracy of $80 \%$. . Zhou and Thuraisingham, [4] focused on finding a robust learning model using spares relevance vector machine ensemble for adversarial learning method. Here using the two real data set one is spam base and another one is web spam. The SVM technique is best for one class 
and adversarial RVM algorithm is best with accuracy is $91 \%$. Zhang et al., [5] introduced a method of STDA (spatial temporal discriminant analysis) is to bring down feature dimensionalities and also improves the appraisal of covariance matrices. It improves average accuracy of $80.8 \%$. Kaper et al., [6], implemented signal from the P300 speller by using the machine learning method. It is more accurate and it demands only 10 electrodes. Wu et al., [7] cast the CSP algorithm. CSP is advice as a generic EEG signal. Zhang et al.,[8], presented that the bayes error can be practically decreased by applying a new spatial filter with lower Rayleigh quotient. Zhang et al.,[9] proposed L1regulerized multiway canonical correlation Analysis for SSVEP-Based BCI in this paper compared with CCA and MCCA, techniques the L1- MCCA achieved higher SSVEP recognition accuracy $80 \%$. Related work are reviewed and presented in the Table 1.

Table 1: Data set and Methods

\begin{tabular}{|c|c|c|c|}
\hline Ref. & Dataset & Method & $\begin{array}{c}\text { Accu- } \\
\text { racy }\end{array}$ \\
\hline 10 & $\begin{array}{c}\text { 64,32 electrodes } \\
8 \text { channels }\end{array}$ & $\begin{array}{c}\text { ICA, } \\
\text { MNE, PCA }\end{array}$ & $85 \%$ \\
\hline 11 & -- & SVM & $91.26 \%$ \\
\hline 12 & $\begin{array}{l}32 \text { electrods } \\
8 \text { channels }\end{array}$ & SVM & $80 \%$ \\
\hline 13 & -- & SVM & $91 \%$ \\
\hline 14 & $\begin{array}{l}16 \text { electrodes, } \\
16 \text { channels }\end{array}$ & ${ }^{1} \mathrm{STDA}$ & $80.8 \%$ \\
\hline 6 & 64 electrodes & SVM & $84.5 \%$ \\
\hline 16 & $\begin{array}{c}60,118 \text { and } 32 \\
\text { channels }\end{array}$ & CSP & $95 \%$ \\
\hline 17 & $\begin{array}{l}59 \text { and } 22 \\
\text { channels. }\end{array}$ & CSP & -- \\
\hline 18 & $\begin{array}{c}105 \text { and } 120 \\
\text { channels, } 27 \text { and } \\
57 \text { electrodes. }\end{array}$ & SVM & $98.9 \%$ \\
\hline 19 & $\begin{array}{l}6 \text { channels and } \\
26 \text { electrodes }\end{array}$ & LVQ, MLP & $70 \%$ \\
\hline 20 & $\begin{array}{c}30 \text { channels and } \\
10 \text { to } 20 \\
\text { electrodes }\end{array}$ & $\begin{array}{c}\text { CCA,MCCA } \\
\text { and }{ }^{2} \mathrm{~L} 1-\mathrm{MCCA}\end{array}$ & $80 \%$ \\
\hline 21 & 9 electrodes & $\begin{array}{c}\text { SVM } \\
\text { Variation RVM }\end{array}$ & $80.68 \%$ \\
\hline 22 & $\begin{array}{l}64 \text { electrodes } \\
\text { and } \\
30 \text { channels }\end{array}$ & ${ }^{3}$ GSBLDA & $90 \%$ \\
\hline
\end{tabular}

$1_{\text {spatial-temporal discriminant analysis, }}{ }^{2} \mathrm{L1}$ regulerized multiway canonical correlation Analysis, 3 group sparse baysian linear discriminator analysis

\section{Methodology}

The proposed methodology includes mainly input data base/data acquisition, pre-processing. The first stage in BCI is data collection. Before parameter extraction is being done, a set of measurements is to be performed. The signals are acquired using the electrode placement or EEG signal can be collected from PhysioBankin website.

In the feature extraction phase, the information that is most relevant for classification is extracted from the raw data. The EEG signal is a complex function of the brain characteristics such as mental stress, emotional state, neurological disorders, e.g., epilepsy, early diagnosis and localization of brain tumors and coma. A good feature extraction scheme should maintain and enhance those features of the input data which make distinct pattern classes.

The proposed methodology is shown in figure 1 .

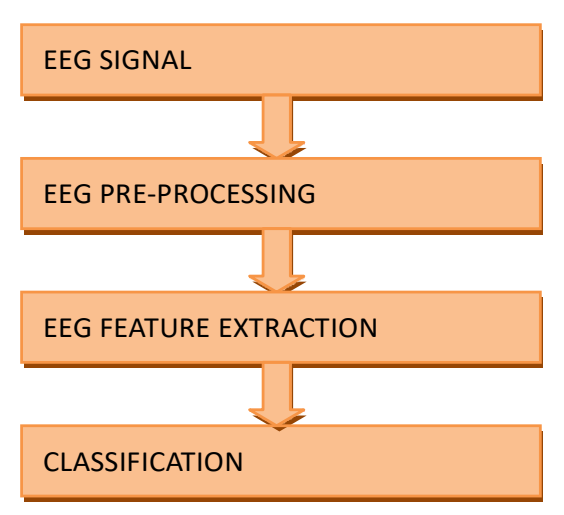

Figure 1: Proposed methodology

In this work, Discrete Wavelet Transform is used to remove the highest frequencies, local information is retained and the image looks like a low resolution version of the full EEG. Wavelets are mathematical functions that cut up data into different frequency components, and then study each component with a resolution matched to its scale. These basis functions are short waves with limited duration, thus the name 'wavelets' is used. The basic functions of the Wavelet Transform are scaled with respect to frequency.

The next most crucial step is classification. All the preceding stages should be designed and tuned to achieve success in the classification 
phase. The operation of the classification step is simplified as being that of transforming a quantitative input data to qualitative output information. Because ANNs can be trained to identify non-linear patterns between input and output values and can solve problems much faster than digital computers, a feed forward neural network trained using Back propagation is used for the recognition of epileptic patterns in EEG signals.

\section{Experimental Results}

In this stage we classify the collected EEG signal either normal or abnormal. In abnormal stage classifying the two Diseases Epilepsy and tumor.

Step 1: The first stage in BCI is data collection. Before parameter extraction is being done, a set of measurements is to be performed. The signals are acquired using the electrode placement or EEG signal can be collected from PhysioBank in www.physionet.org website. The figure 2 shows the original input signal and figure 3 shows the filtered EEG signal.

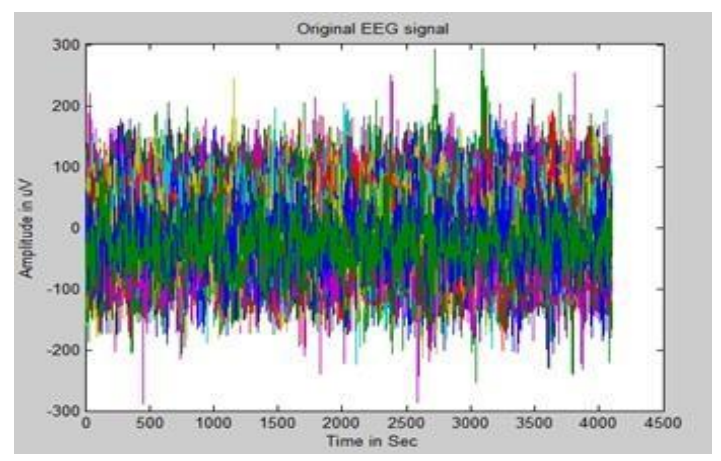

Figure 2: Input of EEG signal

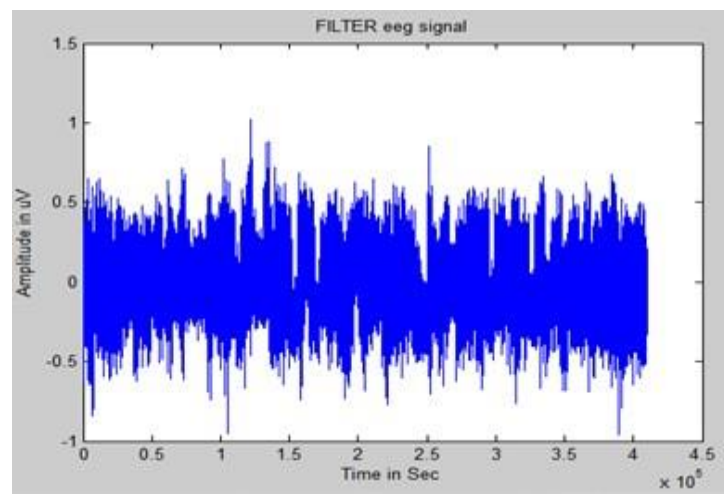

Figure 3: Filtered of EEG signal
Step 2: Pre-processing techniques help to remove unwanted artifacts from the EEG signal and hence improve the signal to noise ratio. A pre-processing block aids in mproving the performance of the system by separating the noise from the actual signal. In proposed method using Recursive least squares (RLS) is an adaptive filter that recursively finds the coefficients that minimize a weighted linear least squares cost function relating to the input signals. Normalizing all the co-efficients and finding the mean to minimize the error. Feature extraction of EEG Signal is shown in figure 4.

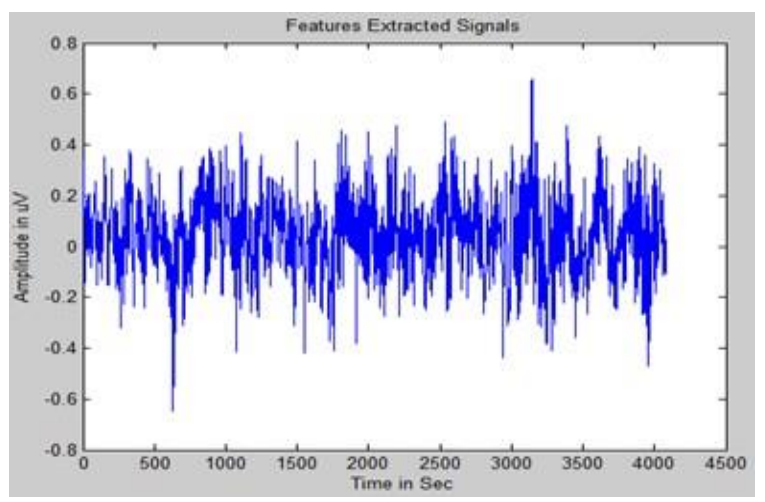

Figure 4: Feature extraction of EEG Signal

Step 3: In the feature extraction phase, the information that is most relevant for classification is extracted from the raw data as shown in figure 5. The EEG signal is a complex function of the brain characteristics such as mental stress, emotional state, neurological disorders, e.g., epilepsy, early diagnosis and localization of brain tumors. In the proposed work, Discrete Wavelet Transform is used.

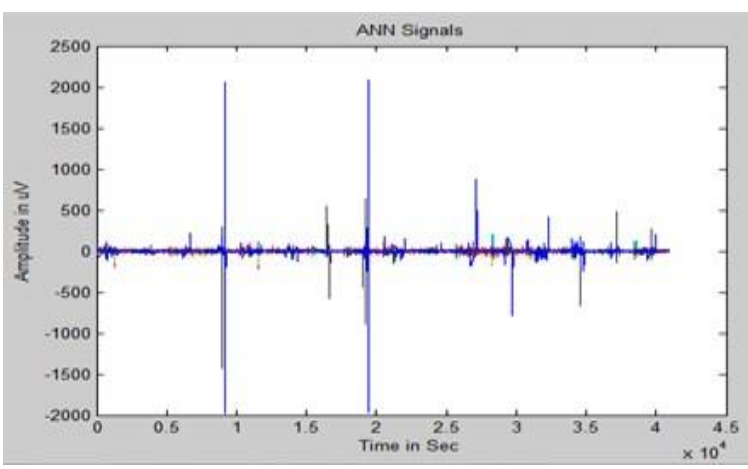

Figure 5: ANN of EEG signal 
Step 4: In this step, a feed forward neural network was trained using Back propagation for the recognition of epileptic patterns in EEG signal. Best validation performance of EEG signal is as shown in figure 6 and figure 7 shows GUI Representation of EEG signal.

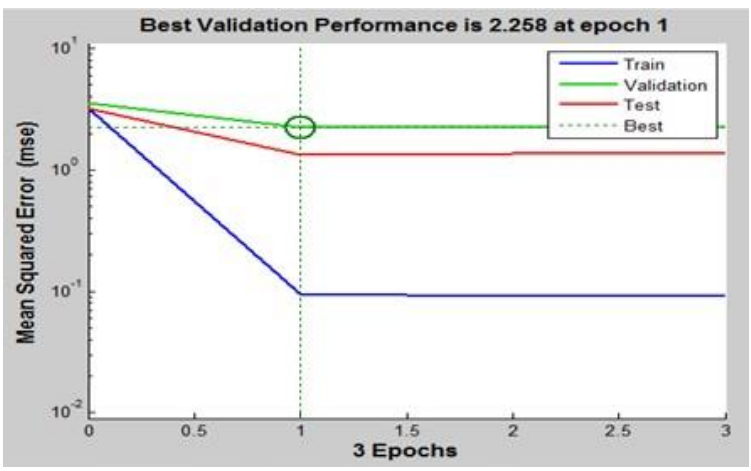

Figure 6: Best validation performance of EEG signal

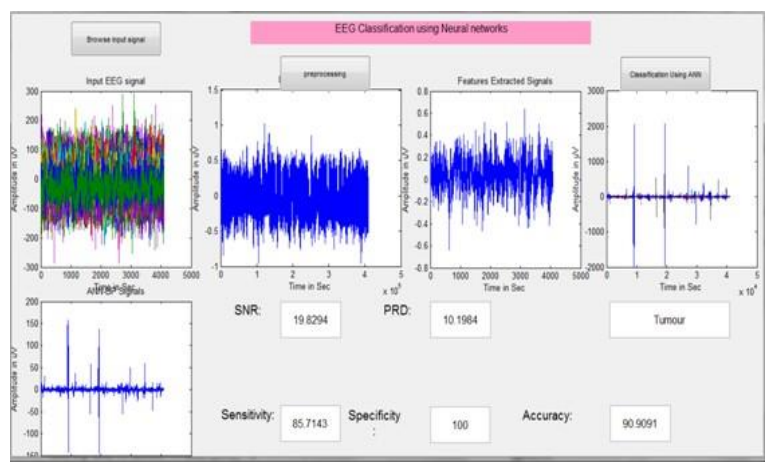

Figure 7: GUI Representation of EEG signal

Step 5: Performance Comparison with respect Normal, Epilipsy and tumor is as shown in figure 8 .

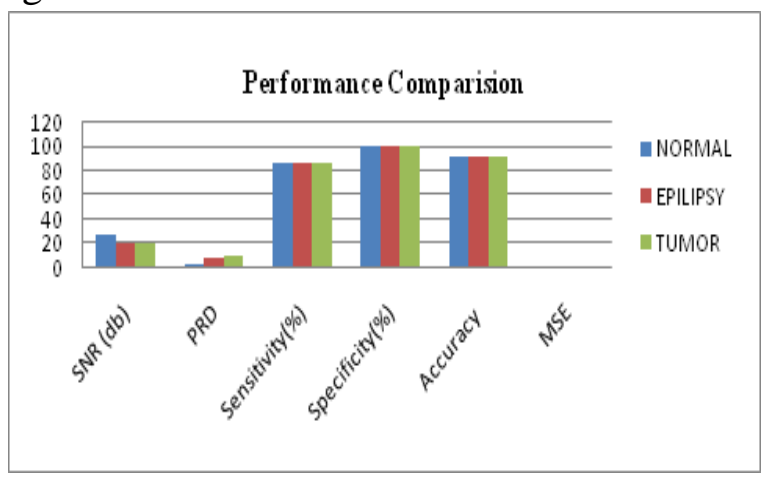

Figure 8: Performance comparison

Step 6: The Statistical Parameters Comparison of the MLBP Classifiers and SVM classifier is as shown in figure 9 and figure 10 and performance comparison is presented in the Table 2 .

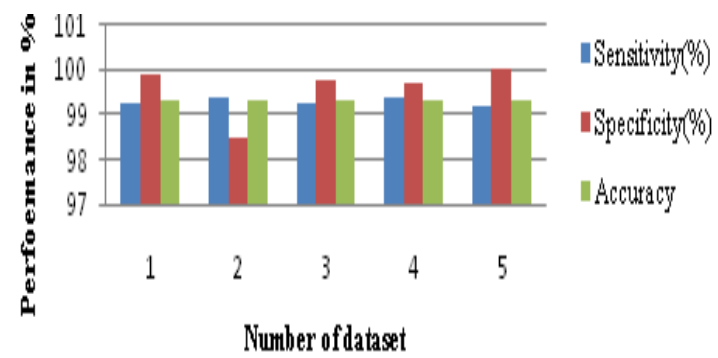

Figure 9: The Statistical Parameters Comparison of the MLBP Classifiers

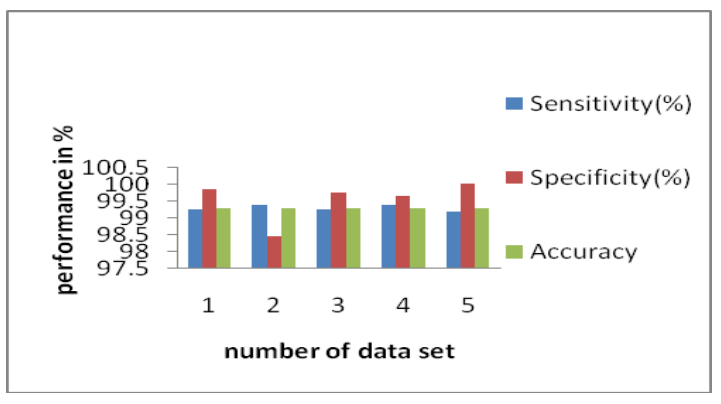

Figure 10: The Statistical Parameters Comparison of the SVM Classifier

Table 2: Performance comparison

\begin{tabular}{|c|c|c|c|c|c|c|}
\hline Diseases case & $\operatorname{SIR}(\mathrm{db})$ & PRD & Sensitrity $(1 \%)$ & Specificity(y) & Accuracy & IISE \\
\hline NORMAL & 27.7172 & 4.1128 & 85.7 & 100 & 90.9 & 1.8249 \\
\hline EPIIPSY & 20.59 & 9.33 & 85.5 & 100 & 90.5 & 2.100 \\
\hline TNDOR & 19.8294 & 10.1984 & 84.9 & 100 & 91.1 & 2.25 \\
\hline
\end{tabular}

\section{Conclusion}

In the proposed work, classifiers for the different diseases are proposed. The performance of the proposed work is measured in terms of different parameters such accuracy, SNR, PRD, sensitivity etc. The artificial neural network (ANN) and MLBP are proposed with a classification accuracy of about $90 \%$ and $95 \%$ respectively which are better in comparison with the related works. 


\section{References}

1. Z. Cashero and C. Anderson, may, 2011 Comparison of EEG blind source separation techniques to improve the classification of P300 trials, Proceedings of the Annual International Conference of the IEEE Engineering in Medicine and Biology Society, Boston, May 2011, 7183-7186.

2. K. Vo, T. Pham, D. N. Nguyen, H. H. Kha and E. Dutkiewicz, Subject-Independent ERP-Based Brain-Computer Interfaces, IEEE Transactions on Neural Systems and Rehabilitation Engineering: Vol. 26, No. 4, April 2018, 719-728.

3.U. Hoffmann, A. Yazdani, J. Vesin and T. Ebrahimi, 2008, Bayesian feature selection applied in a p300 brain-computer interface, Proceedings of the 16th European Signal Processing Conference, Lausanne, Switzerland, August 25-29, 2008, 1-5.

4. Y. Zhou, M. Kantarcioglu and B. Thuraisingham, Sparse Bayesian Adversarial Learning Using Relevance Vector Machine Ensembles, Proceedings of the IEEE $12^{\text {th }}$ International Conference on Data Mining,Brussels, 10-13 Dec 2012, Brussels, Belgium,1206-1211.

5. Y. Zhang, G. Zhou, Q. Zhao, J. Jin, X. Wang, and A. Cichocki, "Spatial-temporal discriminant analysis for ERP-based brain-computer interface," IEEE Transactions on Neural Systems and Rehabilitation Engineering, Vol. 21, Issue 2, March 2013, 233243.

6. Matthias Kaper, Peter Meinicke, U. Grossekathoefer, Thomas Lingner, Helge J. Ritter, BCI competition 2003-data set IIb: support vector machines for the P300 speller paradigm, IEEE Transactions on Biomedical Engineering, Vol. 51, No.6, 2004, 1073-1076.

7. Wei Wu, Zhe Sage Chen, Xiaorong Gao, Yuanqing Li, Emery N Brown, Shangkai GaoProbabilistic Common Spatial Patterns for Multichannel EEG Analysis, IEEE
Transactions on Pattern Analysis and Machine Intelligence, Vol.37, No. 3, 2015, 639-653.

8. H. Zhang, H. Yang, and C. Guan, Bayesian learning for spatial filtering in an EEG-based brain-computer interface, IEEE Transactions on Neural Networks and learning Systems, Vol. 24, No. 7, July 2013, 1049-1060.

9. Y. Zhang, G. Zhou, J. Jin, M. Wang, X. Wang, and A. Cichocki, L1 - regularized multiway canonical correlation analysis for SSVEP-based BCI, IEEE Transactions on Neural Systems and Rehabilitation Engineering vol. 21, No. 6, Nov. 2013, 887896.

10. Z. Cashero and C. Anderson, Comparison of EEG blind source separation techniques to improve the classification of P300 trials, Annual International Conference of the IEEE Engineering in Medicine and Biology Society, Boston, May, 2011, 7183-7186.

11. K. Vo, T. Pham, D. N. Nguyen, H. H. Kha and E. Dutkiewicz, Subject-Independent ERP-Based Brain-Computer Interfaces, IEEE Transactions on Neural Systems and Rehabilitation Engineering, Vol. 26, No. 4, April 2018, 719-728.

12. U. Hoffmann, A. Yazdani, J. Vesin and T. Ebrahimi, Bayesian feature selection applied in a p300 brain-computer interface, 16th European Signal Processing Conference, Lausanne, Switzerland, August 25-29, 2008, 1-5.

13. Y. Zhou, M. Kantarcioglu and B. Thuraisingham, Sparse Bayesian Adversarial Learning Using Relevance Vector Machine Ensembles, IEEE 12th International Conference on Data Mining, Brussels, 2012,1206-1211.

14. Y. Zhang, G. Zhou, Q. Zhao, J. Jin, X. Wang, and A. Cichocki, Spatial-temporal discriminant analysis for ERP-based brain- computer interface, IEEE Trans. Neural System Rehabilitation. Engineering, Vol. 21, No. 2, Mar. 2013, 233-243. 
15. W. Wu, Z. Chen, X. Gao, Y. Li, E. N. Brown, and S. Gao, Probabilistic common spatial patterns for multichannel EEG analysis, IEEE Trans.Pattern Anal. Mach. Intell., Vol. 37, No. 3, Mar. 2015, 639-653.

16. H. Zhang, H. Yang, and C. Guan, Bayesian learning for spatial filtering in an EEG-based brain-computer interface, IEEE Trans. Neural Network Learning System, Vol. 24, No. 7, July 2013, 1049-1060.

17. J. Jin, B. Z. Allison, Y. Zhang, X. Wang, and A. Cichocki, An ERP- based BCI using an oddball paradigm with different faces and reduced errors in critical functions, Int. J. Neural System, Vol. 24, No. 8, 2014, 1450027-1-1450027-14.

18. E. W. Sellers and E. Donchin, A P300based brain-computer interface: Initial tests by ALS patients, Clin Neurophysiol., Vol. 117, No. 3, 2006, 538-548.
19. Y. Zhang, G. Zhou, J. Jin, M. Wang, X. Wang, and A. Cichocki, L1 - regularized multiway canonical correlation analysis for SSVEP-based BCI, IEEE Transaction on Systems and Rehabilitation Engineering, Vol. 21, No. 6, Nov. 2013, 887-896.

20. V. P. Oikonomou, S. Nikolopoulos, P. Petrantonakis and I. Kompatsiaris, Sparse Kernel Machines for motor imagery EEG classification, 40th Annual International Conference of the IEEE Engineering in Medicine and Biology Society (EMBC), Honolulu, Hawaii, 2018, 207-210.

21. T. Yu, Z. Yu, Z. Gu and Y. Li, Grouped Automatic Relevance Determination and Its Application in Channel Selection for P300 BCIs, IEEE Transactions on Neural Systems and Rehabilitation Engineering, Vol. 23, No. 6, Nov. 2015, 1068-1077. 